Ann. Biol. anim. Bioch. Biophys., I970, 10 (3), 369-384.

\title{
UTILISATION DIGESTIVE APPARENTE DES ACIDES GRAS CHEZ LE RAT AXÉNIQUE ET LE RAT HOLOXÉNIQUE ( ${ }^{(1)}$
}

\author{
Y. DEMARne, E. SACQUET, J. FLANZY, H. GARNIER et A.-C. FRANÇOIS \\ Station centrale de Nutrition, \\ Centre national de Recherches zootechniques, 78 -Jouy-en-Josas \\ Institut national de la Recherche agronomique \\ Service des Animaux sans Germes, \\ Centre national de la Recherche scientifique, 91 -Gif-sur-Yvette
}

\section{RF́SUMÉ}

L'utilisation digestive apparente de deux matières grasses saturées contenant 43 p. Ico d'acides gras longs et saturés $\left(\geqslant C_{16}\right)$, mais différant par leur composition en acides gras, a été étudiée chez le Rat axénique et chez le Rat holoxénique. Il ressort de cette étude que le Rat axénique utilise mieux les matières grasses saturées que le Rat holoxénique. L'amélioration de la digestibilité semble être due essentiellement à une meilleure utilisation des acides palmitique $\left(C_{16}\right)$ et stéarique $\left(\mathrm{C}_{18}\right)$.

Parallèlement à ces résultats, on observe une diminution importante de l'excrétion fécale de savons de calcium chez les rats axéniques. Compte tenu du fait que ces composés sont essentiellement synthétisés à partir des acides palmitique et stéarique de la ration, ce phénomène semble expliquer, tout au moins en partie, la meilleure utilisation digestive apparente de ces acides gras en absence de flore intestinale.

\section{INTRODUCTION}

L'influence de la flore intestinale sur l'utilisation digestive des matières grasses et plus particulièrement sur celle des acides gras qui entrent dans leur composition, est encore peu connue. EvRARD et al. (1964) rapportent que des rats axéniques recevant un régime comportant $6 \mathrm{p}$. Ioo de matière grasse insaturée (huile de maîs) excrètent moins de graisses fécales que des rats holoxéniques placés dans les mêmes conditions ( $1,90 \mathrm{mg}$ de graisses fécales par gramme d'aliment ingéré, contre $2,74 \mathrm{mg}$ dans le cas des holoxéniques). Chez le Lapin, Yoshida et al. (I968) rapportent des résultats du même ordre. En l'absence de flore intestinale, l'utilisation digestive

(1) Terminologie proposée par Raibaud et al. (1966). 
apparente de la matière grasse ingérée (huile de maïs) a été améliorée (93,4 p. Ioo contre 89,5 p. Ioo dans le cas du Lapin holoxénique).

Cependant, Tennant et al. (I969) ont montré que le coefficient d'utilisation digestive apparente (CUD) de l'acide oléique ou de la trioléine n'était pas amélioré chez le Rat axénique.

Ces études ont été réalisées en utilisant soit une matière grasse très insaturée (1'huile de maïs contient 85 p. Ioo d'acides gras insaturés), soit avec un acide gras insaturé ou un triglycéride homogène insaturé. Tenant compte du fait que ces matières grasses ou ces acides présentent déjà des CUD apparents très élevés chez l'animal holoxénique, on peut penser qu'il est difficile de mesurer, avec une précision suffisante, l'influence de la flore intestinale en utilisant des matières grasses de ce type. Ainsi, Boyd et EDwards ( 1967 ) ont réalisé une étude de digestibilité comparée entre poulets axéniques et poulets holoxéniques. Ces auteurs utilisèrent des régimes contenant 6 p. Ioo de matières grasses fournies soit sous forme d'huile de maïs (régime insaturé), soit sous forme de suif (régime saturé). Dans le premier cas, les différences enregistrées entre les CUD apparents étaient faibles $(87,6 \mathrm{p}$. Ioo contre 85,6 p. IOo). En revanche, dans le second cas, le CUD apparent du suif a été de 85 , I p. I oo en 1'absence de flore intestinale et de $75 \mathrm{p}$. Ioo en sa présence. Le CUD apparent a donc été amélioré d'une façon plus sensible lorsque les animaux ont ingéré une graisse saturée.

Les expériences dont nous rapportons ici les résultats ont eu pour but de mesurer, chez le Rat, l'influence de la flore intestinale sur l'utilisation digestive de deux matières grasses saturées, de composition différente et définie.

En tenant compte du fait que certains auteurs, comme FLANZY et al. (I968), chez le Porc holoxénique, et FaKAmBi (I970), chez le Rat holoxénique, ont montré que la faible utilisation digestive des acides gras saturés et longs pouvait être due à la formation de savons insolubles dans 1'intestin, nous avons étudié également l'influence de la flore intestinale sur la formation de ces composés.

\section{MATÉRIEL E'T MÉ'THODES}

\section{Aliments}

La composition pondérale du régime qui a été fourni aux animaux est rapportée dans le tableau I. Il contient I 3,3 P. I oo de matières grasses provenant de deux mélanges différents I et II. Les compositions de ces mélanges sont données dans le tableau 2.

TABI,EAU I

Composition pondérale du végime (p. Ioo en poids)

Composition of diet(per cent wt.)

\begin{tabular}{|c|c|}
\hline Caséine. Casein & 17,7 \\
\hline 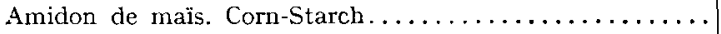 & 56,7 \\
\hline Cellulose. Cellulose $\ldots \ldots \ldots \ldots \ldots \ldots \ldots \ldots \ldots \ldots \ldots$ & 5,4 \\
\hline Sels minéraux. Salt mixture $\ldots \ldots \ldots \ldots \ldots \ldots \ldots$ & 4,8 \\
\hline Mélange vitaminique et acides aminés complémentaires. & \\
\hline Vitamins and complementary amino-acids........... & 2,1 \\
\hline 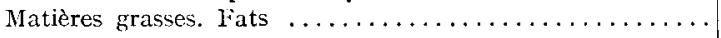 & 13,3 \\
\hline
\end{tabular}


Les matières grasses totales des deux régimes ont été extraites par le mélange chloroformeméthanol $(2 \mathrm{v} / \mathrm{I} \mathrm{v})$. Après saponification, les acides gras extraits ont été méthylés et analysés par chromatographie en phase gazeuse. Le tableau 3 rapporte la composition en acides gras des deux matières grasses. Elles contiennent environ $43 \mathrm{p}$. Ioo d'acides gras longs et saturés $\left(\geqslant \mathrm{C}_{16}\right)$.

\section{TABLEAU 2}

Composition des mélanges de matières grasses (p. Ioo en poids) Component oils (per cent wt.) of fats.

\begin{tabular}{|c|c|c|}
\hline $\begin{array}{l}\text { Huiles } \\
\text { Oils }\end{array}$ & $\begin{array}{l}\text { Régime I } \\
\text { Diet I }\end{array}$ & $\begin{array}{l}\text { Régime II } \\
\text { Diet II }\end{array}$ \\
\hline Huile de Coprah. Coconut oil & 30 & 25 \\
\hline Huile de Colza. Colza oil $\ldots \ldots \ldots \ldots \ldots \ldots$ & 20 & - \\
\hline Huile de Coton. Cotton oil ............... & 10 & 25 \\
\hline Colza hydrogéné. Hydrogenated colza oil & 20 & - \\
\hline Beurre de Cacao. Cocoa butter ........ & 20 & 50 \\
\hline
\end{tabular}

TABLEAU 3

Composition en acides gras des matières grasses des deux régimes (p. Ioo des esters méthyliques dosés)

Component Fatty acids (per cent wt.) of fats.

\begin{tabular}{|c|c|c|c|c|c|c|c|c|c|}
\hline \multirow{2}{*}{$\begin{array}{c}\text { Régimes } \\
\text { Diets }\end{array}$} & \multicolumn{9}{|c|}{$\begin{array}{l}\text { Acides gras } \\
\text { Fatty acids }\end{array}$} \\
\hline & $\mathrm{C}_{12}$ & $C_{14}$ & $C_{16}$ & $C_{18}$ & $C_{18: 1}$ & $C_{18: 2}$ & $\mathrm{C}_{20}$ & $\mathrm{C}_{22}$ & $C_{22: 1}$ \\
\hline I & 15,5 & 6,8 & 12,7 & 16,5 & 15,7 & 9,8 & 4,6 & $8,{ }^{\prime} k$ & 10 \\
\hline II & 15,4 & 6,2 & 23,6 & 19,8 & 29,3 & 5,7 & - & - & - \\
\hline
\end{tabular}

Les aliments ont été stérilisés par passage à l'autoclave ( $120^{\circ} \mathrm{C}$ pendant 20 minutes).

La quantité d'aliments ingérés par chaque rat a été mesurée toutes les semaines. La déshydratation a été déterminée en suivant la perte de poids d'un pellet témoin placé dans les conditions expérimentales. La teneur en matières sèches des refus a été définie après séchage jusqu'à poids constant dans une étuve à circulation à $7^{\circ} \mathrm{C}$.

\section{Animaux}

Les expériences ont été réalisées à l'aide de deux groupes de six rats consanguins, de souche Fisher, âgés d'environ deux mois. L'un des deux groupes était axénique. La croissance des animaux a été régulière, dans les deux groupes et dans les deux expériences. Elle a été de 4 grammes par jour, ce qui est satisfaisant pour des rats de cette souche.

Les rats axéniques ont été maintenus à l'abri des contaminations extérieures dans des isolateurs en film de plastique (isolateurs de Trexler). 


\section{Collecte des fèces et méthodes d'analyses}

Les fèces ont été collectées, pour chaque animal, chaque semaine.

Dans le cas des expériences réalisées avec le régime $\mathrm{I}$, les analyses ont été faites sur 12 collectes, 6 provenant d'animaux axéniques $(A, B, C, D, E, F)$, et 6 provenant d'animaux holoxéniques $(\mathrm{I}, 2,3,4,5,6)$. Dans le cas des expériences réalisées avec le régime II, les collectes des fèces ont été regroupées par lots de 3 rats. Les analyses ont été réalisées sur des échantillons récoltés sur deux semaines consécutives ( $\mathrm{r}$ et 2 ). Nous avons donc analysé dans ce cas 8 lots : 4 provenant d'animaux axéniques $(\operatorname{ABC}(\mathrm{I}), \operatorname{DEF}(\mathrm{I}), \operatorname{ABC}(2), \operatorname{DEF}(2))$, et 4 provenant d'animaux holoxéniques (I. 2. 3. (I), 4. 5. 6. (I), I. 2. 3. (2), 4. 5.6. (2)).

La teneur en matières sèches des fèces a été déterninée après déshydratation jusqu'à poids constant dans une étuve à circulation, à la température de $7^{\circ} \mathrm{C}$. Les fèces ont été ensuite broyées et homogénéisées.

Les lipides fécaux ont été extraits par la méthode de Toullec et al. (1968). Nous avons pu ainsi extraire séparément à froid les lipides libres ou liés aux protéines, d'une part, et ceux des complexes insolubles constitués essentiellement de savons, d'autre part. La première fraction dite "fraction soluble " est extraite par le mélange chloroforme-méthanol selon le principe de la méthode de Folch et al. (1957). Les acides gras de la seconde fraction dite "fraction insoluble" sont libérés de l'extrait précédent par l'acide chlorhydrique, puis extraits par l'éther de pétrole.

Après saponification, les acides gras des différentes fractions ont été extraits puis dosés. La détermination et le dosage des acides gras ont été réalisés par chromatographie en phase gazeuse des esters méthyliques.

\section{RÉSULTATS}

\section{Utilisation digestive apparente globale des matières grasses}

Les valeurs des coefficients d'utilisation digestive apparente sont rapportées dans le tableau 4.

La matière grasse du régime II a été la mieux utilisée par tous les animaux. Cependant, les rats axéniques présentent des CUD apparents supérieurs à ceux des rats holoxéniques. C'est avec le régime II que les différences les plus sensibles ont été enregistrées entre les deux types d'animaux.

\section{Utilisation digestive apparente des acides gras}

Le tableau 5 rapporte les valeurs des CUD apparents des acides gras présents dans les deux régimes.

Dans les deux cas, 1'utilisation apparente des acides gras en $C_{12}$ et $C_{14}$ ne semble pas être améliorée en l'absence de flore intestinale. En revanche, l'utilisation digestive apparente des acides gras saturés en $\mathrm{C}_{16}$ et $\mathrm{C}_{18}$ est supérieure chez les animaux axéniques. C'est avec le régime II que les améliorations sont les plus sensibles. L'utilisation digestive des acides gras insaturés des deux régimes ne paraît pas être influencée par l'absence de flore intestinale.

Pour les acides gras très longs du régime $I\left(\geqslant C_{20}\right)$, vu l'hétérogénéité des résultats, aucune tendance ne peut être dégagée.

\section{Formes d'excrétion fécale des matières grasses}

Le tableau 6 rapporte la quantité de graisses fécales contenues dans la fraction insoluble. Les valeurs moyennes ont été reportées sur la figure r.

L'excrétion de savons insolubles est plus importante en présence de flore intestinale. Cependant, la synthèse de ces composés semble aussi être liée au type de 
FLORE E'T DIGFSTIBILITÉ DES ACIDES GRAS

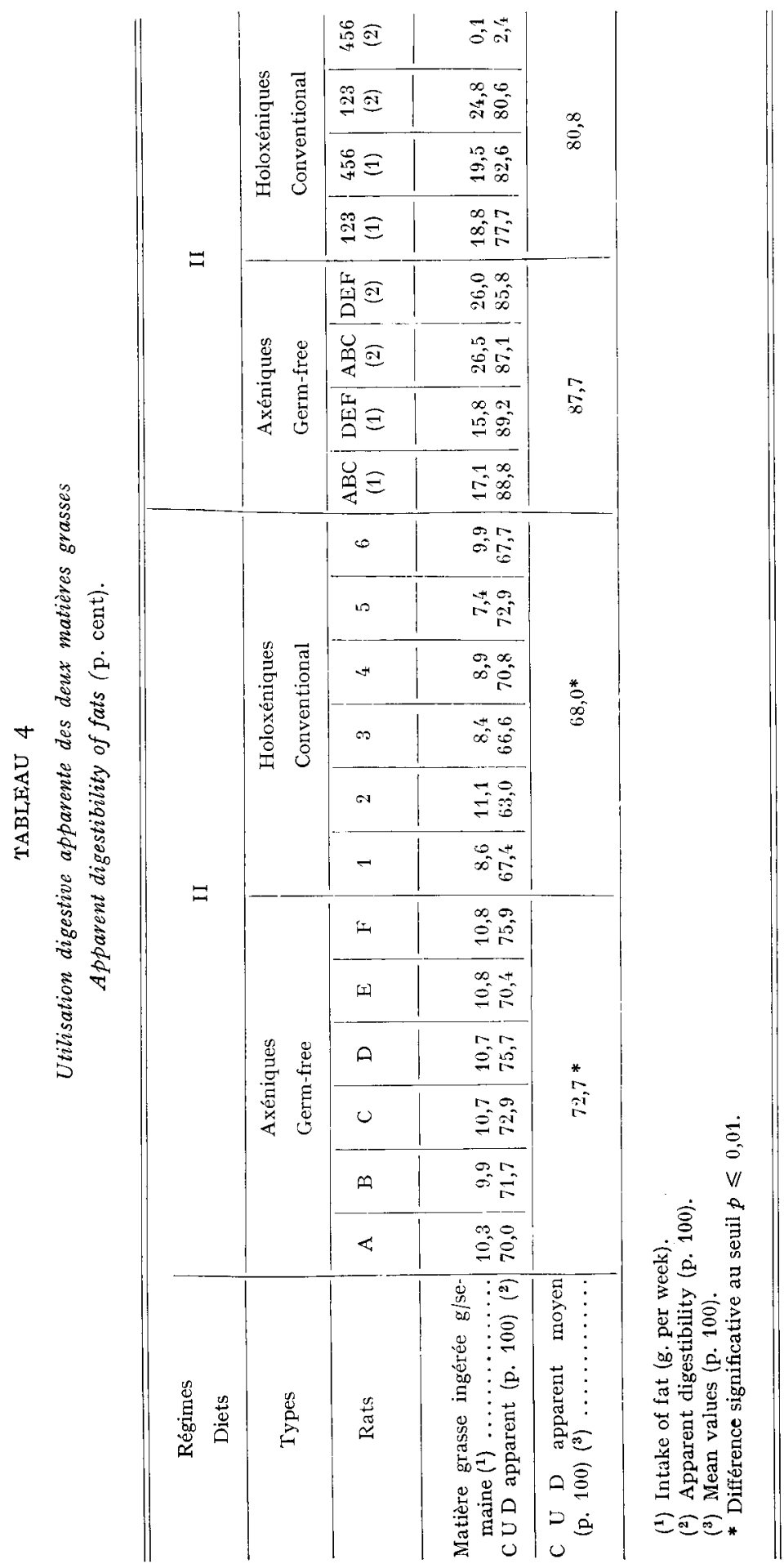


Y. DEMARNE ET COLL.

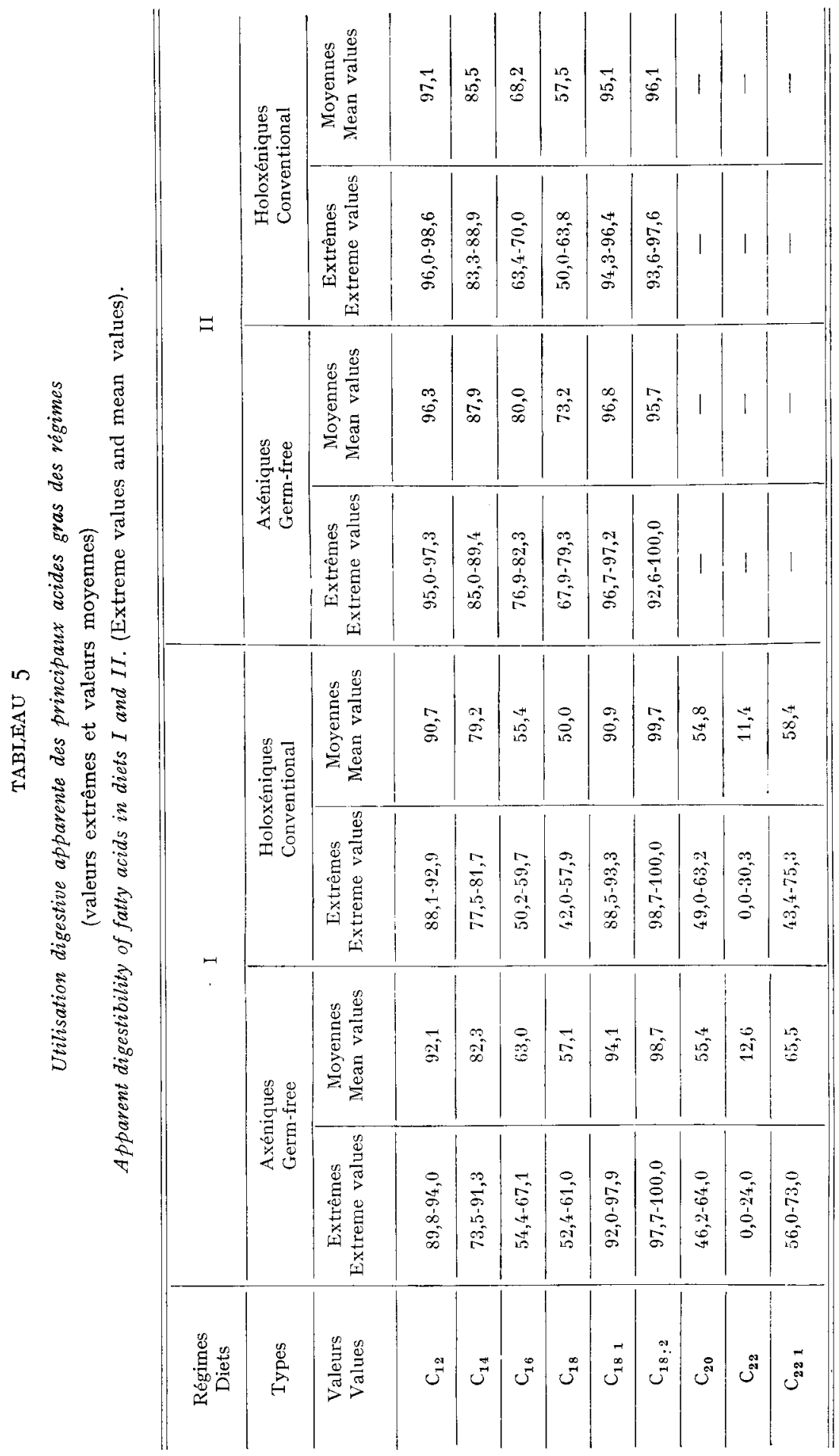


FLORE ET DIGESTIBIL ITTÉ DES ACIDES GRAS

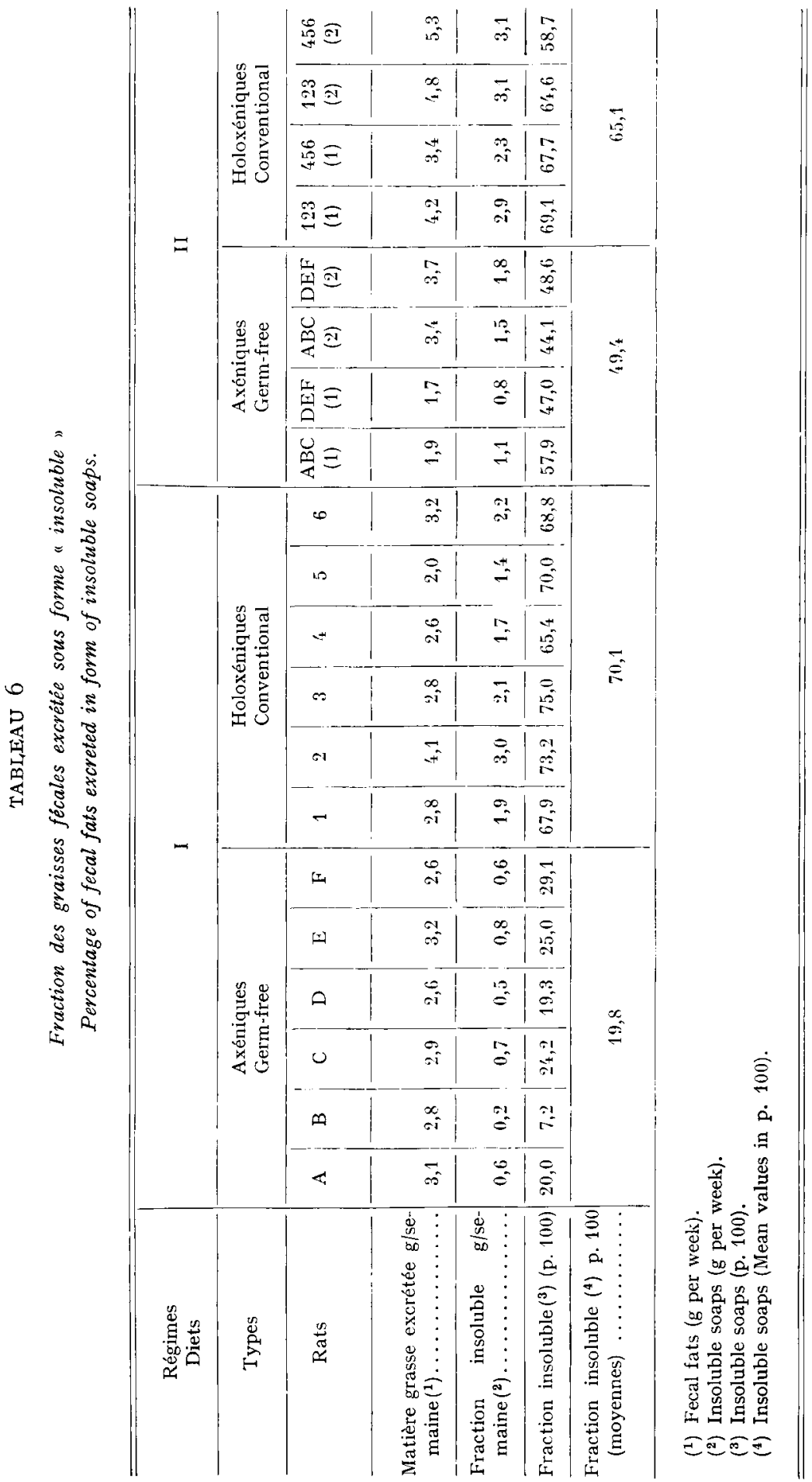




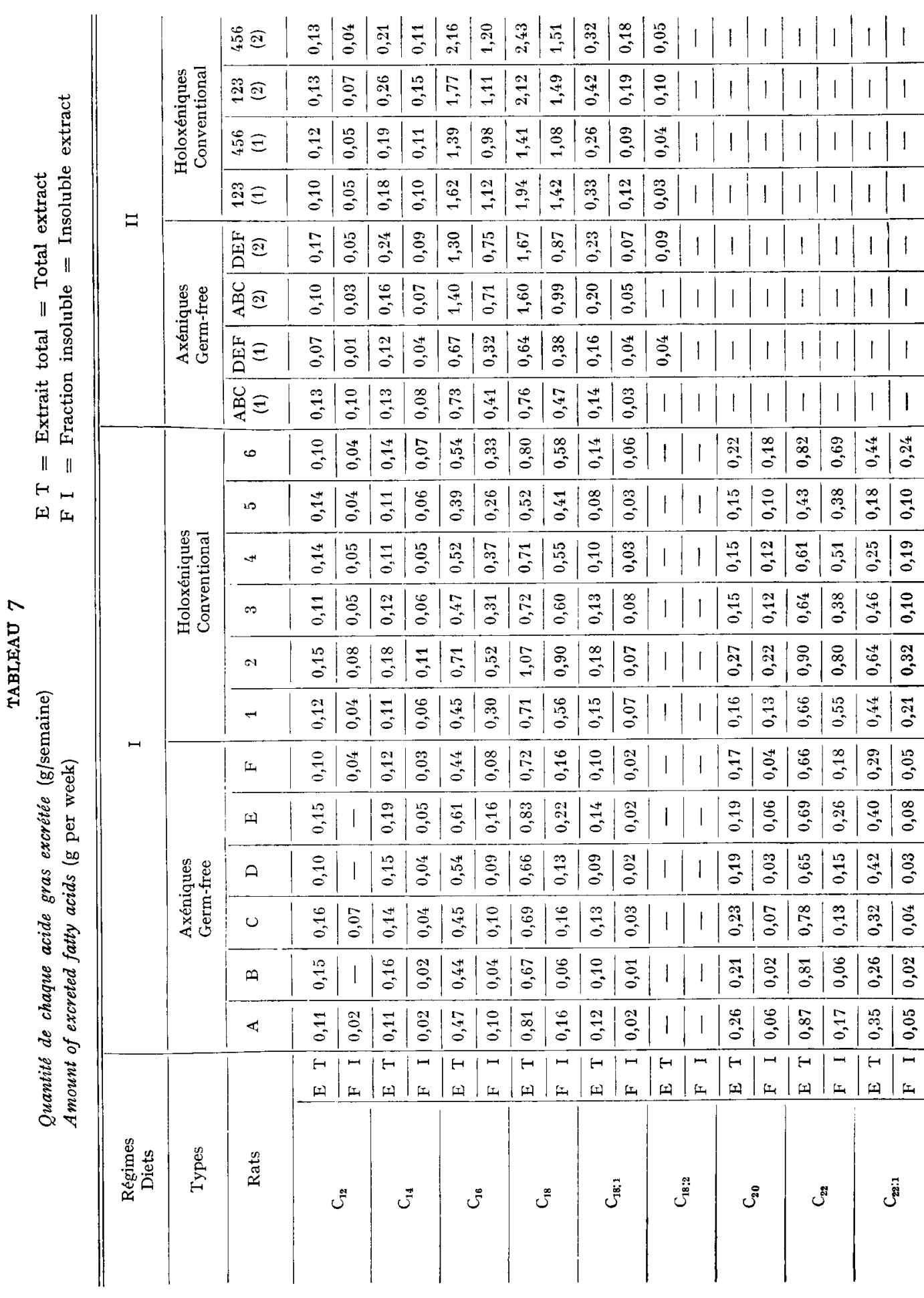




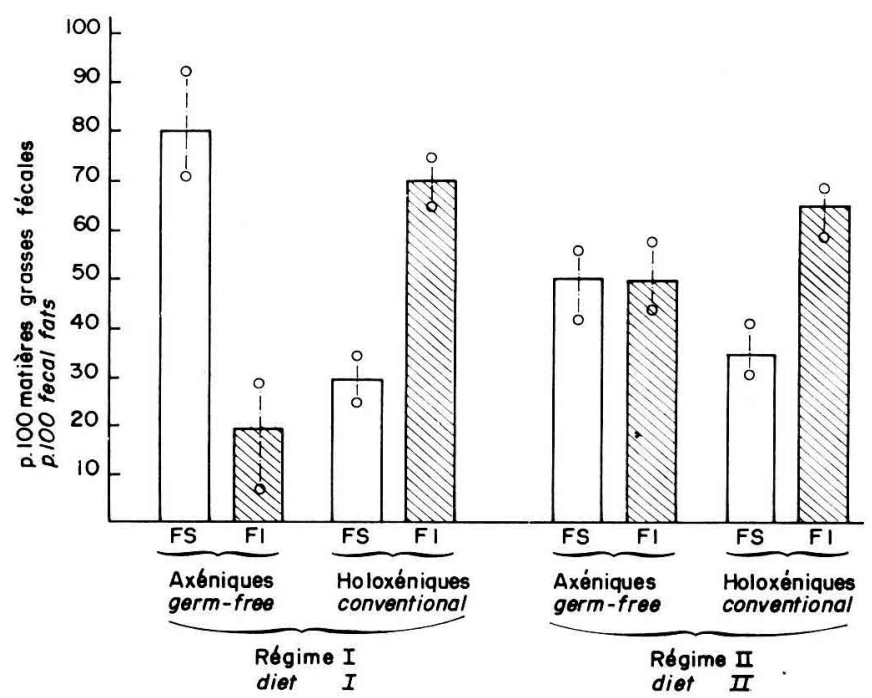

FIc. I. - Kêpartition àes matières grasses fècailes äans les fractions solubie (F $\mathrm{S}$ ) et insoluble (F $\mathrm{I})$. Valeurs extrêmes et moyennes

\section{TABI,EAU 8}

Composition moyenne en acides gras des différentes fractions

(p. Ioo des esters méthyliques dosés)

Component fatty acids of the two different extracts : Soluble Extract (FS), and Insoluble Extract (FI).

\begin{tabular}{|c|c|c|c|c|c|c|c|c|c|c|c|}
\hline \multirow{2}{*}{$\begin{array}{c}\text { Régimes } \\
\text { Diets }\end{array}$} & \multirow[t]{2}{*}{ Rats } & \multirow{2}{*}{$\begin{array}{c}\text { Frac- } \\
\text { tions } \\
\text { Ex- } \\
\text { tracts }\end{array}$} & \multicolumn{9}{|c|}{$\begin{array}{l}\text { Acides gras } \\
\text { Fatty acids }\end{array}$} \\
\hline & & & $\mathrm{C}_{12}$ & $\mathrm{C}_{14}$ & $C_{16}$ & $\mathrm{C}_{18}$ & $C_{18: 1}$ & $C_{18: 2}$ & $\mathrm{C}_{20}$ & $\mathrm{C}_{22}$ & $C_{22: 1}$ \\
\hline \multirow{4}{*}{ I } & Axéniques & $\mathrm{FS}$ & 4,6 & 4,9 & 17,2 & 25 & 3,7 & & 6,8 & 25,2 & 12,6 \\
\hline & Germ-free & FI & 3,3 & 5,6 & 16,8 & 27,6 & 3,4 & & 7,9 & 27,1 & 8,3 \\
\hline & $\begin{array}{l}\text { Holoxé- } \\
\text { niques }\end{array}$ & FS & 9,3 & 6,9 & 17,3 & 17,3 & 9,3 & & 4,8 & 11,4 & 23,7 \\
\hline & $\begin{array}{c}\text { Conven- } \\
\text { tional }\end{array}$ & FI & 2,5 & 3,5 & 17,2 & 29,5 & 2,4 & & 7,3 & 28,7 & 8,9 \\
\hline \multirow{4}{*}{11} & Axéniques & FS & 4,8 & 6,9 & 36,4 & 36,6 & 11,2 & 3,5 & & & \\
\hline & Gcrm-free & $\mathrm{FI}$ & 3,8 & 5,3 & 38,9 & 46,9 & 3,8 & 1,2 & & & \\
\hline & $\begin{array}{l}\text { Holoxé- } \\
\text { niques }\end{array}$ & FS & 4,2 & 5,6 & 39,1 & 36,8 & 12,5 & 1,8 & & & \\
\hline & $\begin{array}{c}\text { conven- } \\
\text { tional }\end{array}$ & FI & 2,2 & 4,7 & 38,9 & 48,4 & 5,0 & 0,9 & & & \\
\hline
\end{tabular}


matières grasses saturées ingérées. C'est, en effet, avec le régime I que les différences entre groupes ont été les plus sensibles.

Le tableau 7 rapporte les quantités globales excrétées de chaque acide gras $(\mathrm{E}, \mathrm{T})$. Nous avons reporté dans le même tableau les quantités de chaque acide gras excrétées sous forme de savons insolubles (F I).

\section{Composition en acides gras des différentes fractions}

Nous avons rapporté dans le tableau 8 la composition moyenne en acides gras des fractions solubles (FS) et insolubles (FI).

Les fractions insolubles paraissent être les plus riches en acides gras longs et saturés $\left(\geqslant \mathrm{C}_{16}\right)$. Dans tous les cas, elles contiennent entre 80 et 87 p. Ioo environ de ces acides.

Les figures 2 et 3 montrent les variations des valeurs moyennes du rapport Fraction insoluble

\section{Extrait total}

$\times$ roo pour chaque acide gras en fonction du régime et selon la présence ou l'absence de flore intestinale.

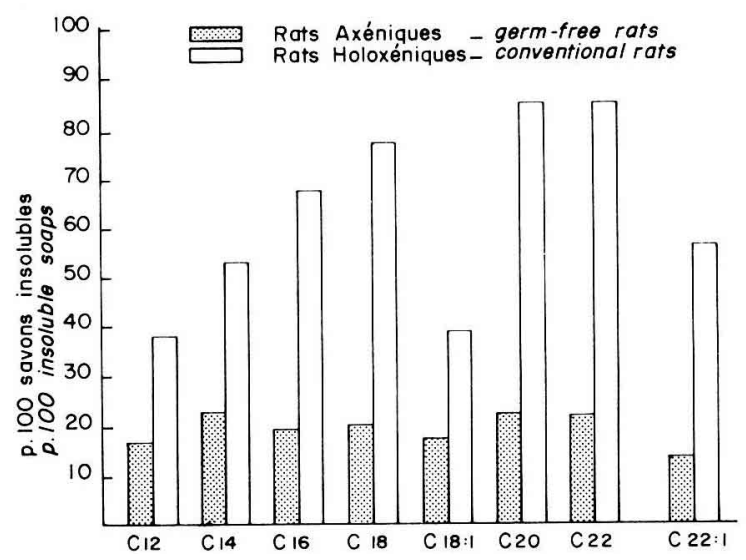

FIG. 2. - Pourcentage de chaque acide gras présent sous forme insoluble dans les fèces (Régime I)

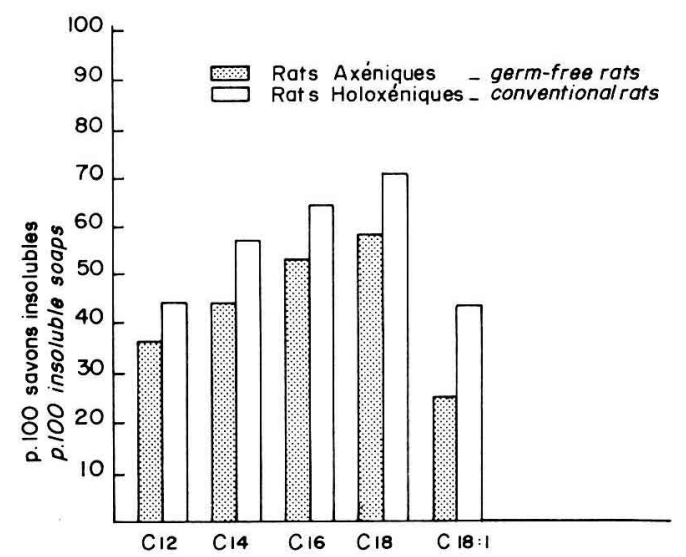

FıG. 3. - Pourcentage de chaque acide gras présent_sous formé insoluble dans les fèces (Régime II) 
Chez les rats holoxéniques ayant ingéré le régime I et chez tous les rats ayant ingéré le régime II, on note que, jusqu'au $\mathrm{C}_{20}$, plus les acides gras saturés sont longs, plus ils ont tendance à être excrétés sous forme insoluble. Dans le cas des rats axéniques ayant ingéré le régime $I$, le phénomène n'apparaît pas.

Cependant, dans tous les cas, on note que, pour chacun des acides gras, une plus grande quantité est excrétée sous forme insoluble en présence de flore intestinale et ce, quelle que soit la matière grasse saturée ingérée.

\section{DISCUSSION ETT CONCLUSIONS}

C'est avec les matières grasses du. régime II que les CUD apparents les plus élevés ont été enregistrés. Ceci peut être expliqué par le fait que les deux matières grasses que nous avons utilisées différaient par leur composition en acides gras. En effet, les matières grasses du régime $I$ contenaient des acides gras très longs $\left(\geqslant C_{20}\right)$, dont on connaît la très faible utilisation digestive.

Les rats axéniques ont mieux utilisé les matières grasses que les rats holoxéniques. La tendance qui se dégage en analysant les résultats obtenus avec la matière grasse I se trouve confirmée par l'analyse des valeurs des CUD obtenus avec le régime II.

Nos résultats confirment ceux de Boyd et EDwards (I967) obtenus chez le Poulet ingérant un régime comportant 6 p. Ioo de suif. En l'absence de flore intestinale, l'utilisation digestive des matières grasses saturées paraît être sensiblement améliorée, surtout lorsque celles-ci ne contiennent pas d'acides gras ayant plus de I 8 atomes de carbone.

Si l'on compare nos résultats àceux de EvRARD et al. (I964) ou de YoshidA et al. (I 968), on note que les améliorations des CUD apparents obtenus avec des matières grasses saturées du type II $(87,7 \mathrm{p}$. I 00 contre $80,8 \mathrm{p}$. Ioo en présence d'une flore intestinale) sont supérieures à celles obtenues avec des matières grasses insaturées. I1 semble donc que les résultats_obtenus par Boyd et EDwards chez le Poulet (I967) soient confirmés chez le Rat. Les CUD sont améliorés d'une façon plus importante lorsque les animaux ingèrent des graisses saturées comme la matière grasse II ou le suif.

Ces données sont en relation avec les résultats que nous enregistrons avec nos deux régimes, lorsque nous"analysons les CUD apparents de chaque acide gras. $L_{1}$ 'utilisation digestive des acides gras en $C_{12}$ et $C_{14}$ ne semble pas être influencée par l'état axénique. Comme Boyd et Edwards (I967) et TenNant et al. (I969) l'ont précédemment souligné, la flore intestinale ne semble pas influencer l'utilisation digestive des acides gras insaturés. Il apparaît que l'influence se situe surtout au niveau des acides gras saturés en $\mathrm{C}_{16}$ et $\mathrm{C}_{18}$ (palmitique et stéarique). Pour les acides gras saturés très longs, l'influence semble nulle, mais les résultats, vu leur hétérogénéité, sont difficiles à interpréter.

Ceci explique bien le fait que les matières grasses insaturées, comme l'huile de maïs, qui ne contiennent qu'une faible quantité d'acides palmitique et stéarique, présentent des CUD apparents très peu influencés par la flore intestinale. Il en serait de même pour les matières grasses contenant peu d'acides gras saturés en $C_{\mathbf{1 6}}$ et $\mathrm{C}_{\mathbf{1 8}}$, mais beaucoup d'acides gras très longs $\left(\geqslant \mathrm{C}_{20}\right.$ ) (régime I par exemple). 
Comment peut-on expliquer un tel phénomène ? D'une part, de nombreux auteurs (CARRol, et RICHARDS, I958 ; RICHARds et CARROLL, I959; FleisChMANN et al., I966 ; PeTTERSON, I964 ; YACOWITZ et al., I967 ; FlaNZY et al., I968 ; FAKAMBI, I970) établissent une relation entre excrétion fécale d'acides gras et de calcium. D'autre part, TOULLEC et al. (Ig68) ont montré que, in vitro, l'addition de chlorure de calcium à une solution équimolaire d'acide palmitique, stéarique, oléique et linoléique donnait lieu à la formation d'un précipité de savons de calcium formé pour 97,5 p. Ioo par les acides saturés du mélange. Plus récemment, FiAnzy et al. (I968), sur le Porc, et FaKambi (I970), sur le Rat, ont montré que la faible utilisation digestive des matières grasses était en relation avec la formation de savons de calcium à partir des acides palmitique et stéarique ingérés. Ce dernier auteur, en particulier, a observé que lorsque l'on abaisse la teneur en calcium de la ration, le CUD apparent global des matières grasses ingérées est amélioré et, parallèlement, on observe une diminution de la teneur en savons insolubles des fèces, ainsi qu'une augmentation du CUD apparent des acides palmitique et stéarique.

On peut donc penser que la meilleure utilisation digestive des matières grasses enregistrée chez le Rat axénique s'explique par la diminution de la formation de savons insolubles au niveau intestinal. Celle-ci est très nette pour les acides palmitique et stéarique et n'apparaît pas clairement avec les acides gras insaturés. Il faudrait rapprocher la diminution de l'excrétion fécale de savons insolubles du fait que le Rat axénique utilise mieux le calcium alimentaire que le Rat holoxénique (Garnier et Sacquet, I969). Toutefois, nous ne connaissons pas les valeurs des CUD apparents du calcium dans le cas de nos expériences.

Il est possible que la synthèse de savons de calcium dans l'intestin du Rat axénique soit limitée par le fait que la quantité d'ions $\mathrm{Ca}^{++}$disponibles y est plus faible que dans celui du Rat holoxénique. Dans ce cas, chez le Rat axénique, puisque les deux régimes que nous avons utilisés contiennent la même quantité de calcium et d'acides gras longs et saturés $\left(\geqslant \mathrm{C}_{16}\right)$, une quantité relativement constante d'acides gras doit être précipitée sous forme insoluble. En étudiant le rapport :

$\frac{\text { savons insolubles excrétés }(\mathrm{g})}{\text { matière grasse ingérée }(\mathrm{g})} \times$ Ioo chez le Rat axénique, on devrait donc obtenir, avec les deux régimes, des valeurs identiques. Les valeurs moyennes ont été reportées sur la figure 4 .

On notera que le rapport reste, en effet, relativement constant pour les rats axéniques, qu'ils ingèrent le régime I ou le régime II. En revanche, chez les rats holoxéniques, les valeurs varient selon le type de matière grasse saturée ingérée. Avec la matière grasse I, le rapport est élevé (présence d'acides gras très longs $\left(\geqslant \mathrm{C}_{20}\right)$. Il s'abaisse fortement lorsque les rats ingèrent le régime II, mais reste toujours supérieur aux valeurs enregistrées avec les rats axéniques (22 et I 2 p. Ioo contre $6 \mathrm{p}$. IOo).

On notera, cependant, que les CUD apparents des acides gras saturés très longs $\left(\geqslant \mathrm{C}_{20}\right)$ du régime I ne paraissent pas être influencés par la flore intestinale. La formation de savons insolubles à partir de ces acides semble pourtant fortement diminuée en l'absence de flore intestinale. Dans ce cas précis, on peut penser que les facteurs limitant l'utilisation digestive sont d'ordre physique (point de fusion, solubilité micellaire, encombrement stérique, autant de facteurs liés entre eux).

La meilleure utilisation digestive du calcium et des acides gras saturés chez le 
Rat axénique est peut-être également en rapport avec la quantité de sels biliaires présents au niveau de l'intestin grêle.

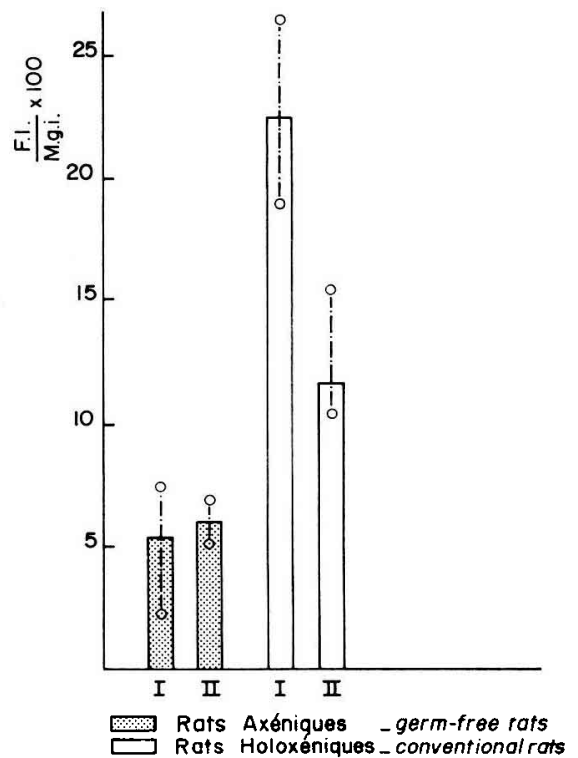

FIG. 4. - Représentation graphique des valeurs moyennes du rapport

Fraction insoluble excrétée (F I)/Matières grasses ingérées (M.Y. $\mathrm{F}$. .) selon les régimes $I$ et $I I$ ( $\times$ Ioo)

Bien que nos connaissances concernant les sels biliaires du Rat axénique soient encore fragmentaires, il est très vraisemblable que la quantité de sels biliaires présents au niveau de l'intestin grêle est supérieure chez le Rat axénique à ce qu'elle est chez le Rat holoxénique. La quantité totale de taurocholate mesurée par des méthodes précédemment décrites (SACQUE⿰氵 et al., I968) au niveau de l'intestin grêle et rapportée à roo grammes de poids vif de l'animal se répartit de la façon suivante :

\section{TABLEAU 9}

Quantité de taurocholate dans la lumière et la paroi intestinale

(micromoles p. $100 \mathrm{~g}$ de poids vif).

Taurocholic acid in the lumen and intestinal wall of germ-free and conventional rats (micromoles p. cent $g$ of living rats).

\begin{tabular}{c|c|c}
\hline \multicolumn{1}{c}{ Rat Fisher } & $\begin{array}{c}\text { Nombre de rats } \\
\text { Number of rats }\end{array}$ & $\begin{array}{c}\text { Quantité de taurolate } \\
\text { Taurocholic acid }\end{array}$ \\
- Axénique. Germ-free ........... & 10 & 16,8 \\
- Axénique cacectomisé. Germ-free \\
and cæcectomized ..........
\end{tabular}

KELLOG et WOSTMANN (I969), utilisant la chromatographie en phase gazeuse, observent que, dans les fèces du Rat axénique, 1'acide cholique n'est pas l'acide 
biliaire quantitativement le plus important, contrairement à ce qui est observé chez le Rat holoxénique. Il en est de même dans la bile (KELLOGG, I969).

S'il en est ainsi, il est possible de spéculer que la quantité d'acides biliaires au niveau de l'intestin grêle est, a fortiori, beaucoup plus importante chez le Rat axénique. Or, le rôle des sels biliaires dans la solubilisation des sels de calcium et de leur absorption a été décrit par WEBLING et HOLDSWORTH (r966) et une diminution de la concentration de sels biliaires s'accompagne d'une diminution de la solubilisation et de l'absorption de ces sels de calcium.

De nombreux travaux ont été consacrés au rôle que jouent les sels biliaires dans la digestion des lipides (HoFmanN, I963; HoFMAnN et BORGSTRÖM, I963; HOFMANN, I966; DAwSON, I967). Bien que le mode d'action de ces sels biliaires sur la digestion des lipides demeure imparfaitement connu, il est hors de doute que ce rôle est considérable et qu'une diminution de la concentration en sels biliaires aboutit à une malabsorption des lipides (DIETSCHY, I967). Récemment, BADLEY et al. (I969) a introduit la notion de "concentration physiologique critique " au-dessous de laquelle apparaît le symptôme de stéatorrhée.

En revanche, dans quelle mesure une augmentation de la concentration en sels biliaires, au-dessus des valeurs observées chez le Rat holoxénique, améliore-i-elle la digestibilité des lipides et des sels de calcium? Ceci n'est pas connu. Si l'on considère que, d'une part, l'augmentation de la digestibilité des lipides chez le Rat axénique est de faible amplitude et que, d'autre part, il est difficile d'augmenter artifciellement la concentration de l'intestin grêle en acides biliaires sans obtenir des effets secondaires, cette notion apparaît difficilement accessible à l'expérimentation.

Il convient de noter, en outre, que la flore intestinale du Rat, encore assez mal connue, présente de nombreuses variations d'un élevage à un autre. Il serait certainement intéressant d'étudier l'effet de diverses souches sur l'utilisation digestive des matières grasses. L'emploi de rats gnotoxéniques serait un moyen d'y parvenir. On pourrait plus particulièrement mesurer, chez des rats à flore contrôlée, l'influence d'une ou plusieurs'souches sur la formation des savons de calcium pendant le transit intestina1. Les travaux de CoLE et Boyd ( 1967 ) sont, sur ce plan, démonstratifs. Chez le Poulet gnotoxénique, ces auteurs ont montré que deux souches bactériennes (Clostridium welchii et Streptococcus faecalis) semblent avoir une action directe sur l'utilisation digestive des acides palmitique et stéarique. Cette action paraît être accentuée lorsque les deux souches sont associées. En effet, si les tutilisations digestives apparentes des acides palmitique et stéarique sont respectivement de 7o p. IOO et de $5^{2} \mathrm{p}$. Ioo chez l'axénique, elles tombent à $6 \mathrm{I} \mathrm{p}$. Ioo et à $4 \mathrm{I} \mathrm{p}$. Ioo chez le Poulet gnotoxénique contaminé avec les deux souches. A notre connaissance, aucun travail de ce genre n'a encore été entrepris chez le Rat.

La flore intestinale semble donc jouer un rôle certain dans l'utilisation digestive des matières grasses. Son action paraît se situer essentiellement au niveau de l'utilisation digestive des acides gras saturés en $\mathrm{C}_{16}$ et $\mathrm{C}_{18}$. Fille intervient efficacement dans les phénomènes de synthèse des savons insolubles, formés principalement à partir de ces deux acides. Son mode d'action directe reste cependant inconnu et de nombreux travaux paraissent encore être nécessaires pour élucider l'ensemble de ces phénomènes, dont l'abord reste souvent difficile. 


\section{SUMMARY}

\section{APPARENT DIGESTIBILITY OF FATTY ACIDS IN GERM-FREE AND CONVENTIONAI, RATS}

Apparent digestibility of two saturated fats, containing 43 p. Ioo long-chain saturated fatty acids $\left(\geqslant \mathrm{C}_{16}\right.$ ), but differing in their fatty acid composition, was studied in germ-free and conventional rats. It emerged from this study that the germ-free rats used saturated fats better than did the conventional. The improvement in digestion seemed essentially to be due to better utilization of palmitic $\left(\mathrm{C}_{16}\right)$ and stearic $\left(\mathrm{C}_{18}\right)$ acid.

In parallel with these results, a large reduction of excretion of calcium soaps in faeces of the germ-free rats was seen. In view of the fact that these compounds are essentially synthesized from the palmitic and stearic acids in the diet, the phenomenon seems to explain at least in part the better apparent digestibility of these fatty acids when there is no intestinal flora.

\section{RÉFÉRENCES BIBLIOGRAPHIQUES}

Badley B. W. B., Murphy G. M., Bouchier I. A. D., Ig69. Intraluminal bile salt deficiency in the pathogenesis of steatorrhea. Lancet, $2,400-402$.

Boyd F. M., Edwards Jr. H. M., r967. Fat absorption by germ-free chicks. Poultry Sci., 46, I48I 1483 .

Carroll K. K., Richards J. F., I958. Factors affecting digestibility of fatty acids in the rat. J. Nutr., 64, 4II-424.

Cole Jr. J. R., Boyd F. M., r967. Fat absorption from the small intestine of gnotobiotic chicks. Appl. Microbiol., 15, I229-1234.

Dawson A. M., I967. Absorption of fats. Brit. med. Bull., 23, 247-25I.

Dietscry J. M., 1967, Effects of bile salts on intermediate metabolism of the intestinal mucosa. Fedn. Proc., 26, I $589-1598$.

Jvrard E., Hoet P. P., Eyssen H., Charlier H., Sacquet E., ig64. Fecal lipids in germ-free and conventional rats. Brit. J. Exp. Pathol, 45, 409-4I4.

FакамBi L., 1970. Mise en évidence de l'excrétion fécale de savons de calcium chez le Rat st son incidence sur l'utilisation digestive des acides gras, du calcium et du phosphore. Thèse $3^{\mathrm{e}}$ cycle, Fac. Sci. Paris.

Flanzy J., RÉrat A., François A. C., I968. Étude de l'utilisation digestive des acides gras chez le Porc. Ann. Biol. anim. Bioch. Biophys., 8, 537-548.

lileischmann A. I, Yakowitz H., Hayton T., Bierenbaum M. L., I966. Effect of dietary calcium upon lipid metabolism in mature male rats fed beef tallow. $J$. Nutr., 88. 255-260.

Folch J., Less M., Sloane-Stanley G. H., 1957. A simple method for the isolation and purification of total lipids from animal tissues. J. biol. Chem., 31, 44I-444.

Garnier H., Sacquet E., rg69. Absorption apparente et rétention du sodium, du potassium, du calcium et du phosphore chez le Rat axénique et chez le Rat holoxénique. C. R. Acad. Sci. Paris, Série D, 269, 379-382.

Hofman A. F., I963. The behavior and solubility of monoglycerides in dilute, micellar bile salt solution. Biochim. biophys. Acta, 70, 306-3 I6.

Hofmann A. F., Ig66. A physiochemical approach to the intraluminal phase of fat absorption. Gastroenterology, 50, 56-64.

HofmanN A. F., Borgström B., I963. Hydrolysis of long chain monoglycetides in micellar solution by pancreatic lipase. Bioch. biophys. Acta, 70, 3I7-33I.

KenLogg T. F., I 969 . Communication personnelle à E. Sacquet.

Kellogg T. F., Wostmann B. S., I969. lecal neutral steroids and bile acids from germ-free rats. J. Lipid Res., 10, 495-503.

l'ETTERSON, I964. Inverkau av hög kalciu mgiva pa totalfettets och de enokilda fettsyrornas smältbarket hos växaude svin. Lantbrukögskolaus meddelanden, Série A, 16, I2 p.

Raibaud P., Dickinson A. B., Sacquet E., Charlier H., Mocquot G., ig66. La microflore du tube digestif du Rat. Ann. Inst. Pasteur, 111, I93-2 I0.

Richards J. F., CARRoll K. K., I959. Studies on calcium phosphate fatty acid salts occuring in fecal lipids. Can. J. Biochem. Physiol., 37, 725-730. 
Sacquet E., Garnier H., Raibaud P., Eyssen H., I968. Étiologie bactérienne de la stéatorrlhée observée chez le Rat porteur d'un cul-de-sac intestinal. Déconjugaison de l'acide taurocholique. $C . R$. Acad. Sci., Paris, Série D, 267, 2238-2240.

Tennant B., Reina-Guerra M., Harrold D., Goldman M., I969. Influence of microotganisms on intestinal absorption : oleic acid ${ }^{131}$ I. Absorption by germ-free and conventionalized rats. $J$. Nutr., 97, 67-69.

Toullec R., Flanzy J., Rigaud J., I968. Dosage des lipides des fèces, Ann. Biol. anim. Bioch. Biophys., 8, 28I-289.

Webling D. D'A., Holdsworth E. S., 1966. Bile salts and calcium absorption. Biochem. J., 100, 652-66o.

Yacowitz H., Fleischman A. I., Amsden R. T., Bierenbaum M. L., 1967. Effects of dietary calcium upon lipid metabolism in rats fed saturated or unsaturated fat. J. Nutr., 82, 389-392.

Yoshida T., Pleasants J. R., Reddy B. S., 1968. Efficiency of digestion in germ-free and conventional rabbits. Br.J. Nutr., 22, 723-737. 